\title{
Gestores municipais do sistema único de saúde: dificuldades na gestão e no planejamento das ações de saúde
}

Municipal managers of the unique health system:

difficulties in the management and planning of health actions

Sandra Maria Mello

\section{RESUMO}

Trata-se de uma pesquisa descritiva com abordagem qualitativa realizada junto aos gestores de municípios da região de abrangência da $12^{\mathrm{a}}$ Coordenadoria Regional de Saúde do Rio Grande do Sul. Os objetivos foram identificar as dificuldades encontradas pelos gestores municipais de saúde no desenvolvimento de suas funções e verificar o conhecimento destes acerca dos instrumentos de planejamento em saúde. Após o processo de análise de conteúdo, os resultados foram agrupados em duas categorias temáticas, quais sejam: dificuldades na gestão e planejamento das ações em saúde. Conclui-se que nem todos os gestores estão preparados para utilizar ou não conhecem, suficientemente, os instrumentos de planejamento e, portanto, não apresentam condições efetivas para administrar de maneira eficaz os recursos disponíveis. Desta forma, torna-se indispensável que os gestores e futuros gestores passem por processos de capacitação e educação continuada a fim de que possam exercer suas atividades com maior fluidez e êxito.

Palavras-chave: Gestor de Saúde. Sistema Único de Saúde. Regionalização. Saúde Pública.

1 Instituto Federal Farroupilha - Campus Santo Ângelo | sandra.cardoso@iffarroupilha.edu.br 


\section{Gestores municipais do sistema único de saúde: \\ dificuldades na gestão e no planejamento das ações de saúde}

Municipal managers of the unique health system:

difficulties in the management and planning of health actions

\section{ABSTRACT}

This is a descriptive research with a qualitative approach conducted with the managers of municipalities in the region covered by the 12th Regional Health Coordination of Rio Grande do Sul. The objectives were to identify the difficulties encountered by municipal health managers in the development of their health functions and verify their knowledge about health planning instruments. After the content analysis process, the results were grouped into two thematic categories, namely: difficulties in the management and planning of health actions. It is concluded that not all managers are prepared to use or do not know enough about planning tools and, therefore, do not have effective conditions to effectively manage available resources. Thus, it is essential that managers and future managers go through processes of training and continuing education so that they can perform their activities with greater fluidity and success.

Keywords: Health Manager. Health Unic System. Regionalization. Public health. 


\section{Introdução}

No Sistema Único de Saúde (SUS), a atividade de gestão implica em coordenar, articular, negociar, planejar, acompanhar, controlar, avaliar e auditar o sistema de saúde em nível municipal, estadual e nacional. Esses atos têm como objetivo o bem comum e seguem os preceitos do direito e da moral (BRASIL, 2013).

Independentemente da gerência dos estabelecimentos prestadores de serviços ser estatal ou privada, a gestão de todo o sistema municipal é, necessariamente, da competência do poder público e exclusiva desta esfera de governo, respeitadas as atribuições dos respectivos Conselhos e de outras diferentes instâncias de poder. São, portanto, gestores do SUS os Secretários Municipais e Estaduais de Saúde e o Ministro da Saúde, que representam, respectivamente, os governos Municipais, Estaduais e o Federal (BRASIL, 2006).

Os secretários de saúde devem também buscar, na medida do possível, superar as disputas político-partidárias da campanha eleitoral e aproveitar as potencialidades e saberes dos profissionais comprometidos com o SUS para planejar a sua gestão, uma vez que, o SUS é um sistema suprapartidário (BRASIL, 2013). É muito importante que o secretário possua uma boa sustentabilidade e representação política no governo municipal, porém, a gestão do SUS não possui apenas a perspectiva política. O conhecimento técnico em saúde pública e de planejamento estratégico são as bases para uma boa governabilidade. 0 gestor deve permanentemente se manter atualizado acerca das questões técnicas de maior relevância.

Em detrimento das inúmeras mudanças que aconteceram na Saúde Pública, houve e ainda há a necessidade de se aprofundar os estudos e conhecimentos que envolvem o processo de gestão pública em saúde. É necessário que os gestores estejam preparados e qualificados para entender os problemas e os desafios que se apresentam no processo de gestão em saúde (MARTINS; WACLAWOVSKY, 2015).

Alguns gestores de municípios de diferentes portes acabam "conformando-se" em manter o instituído, tendo em vista as enormes dificuldades para operar as mudanças nos processos de produção do cuidado em saúde. 0 modelo hegemônico tem sido forte o suficiente para resistir às tentativas de reforma das práticas sanitárias (CAMPOS, 2000).

O SUS tem, entre as suas fraquezas, as dificuldades referentes à insuficiência de financiamento e de apoio técnico. O gestor municipal deve ter clareza de suas limitações e buscar superá-las de forma responsável. Isto pode significar o espaço entre o sucesso e a reprovação do projeto de saúde e principalmente no crescimento ou não da qualidade de vida e saúde da população de seu município.

Assim, destaca-se a importância desse estudo, tendo em vista a dificuldade que muitos gestores encontram ao assumir a secretaria municipal de saúde, visto que, para alguns trata-se de uma missão profissional nova e, para outros, representa a continuidade de uma agenda com muitas potencialidades e limitações. Frente ao exposto, o presente trabalho tem como objetivos identificar as dificuldades encontradas pelos gestores municipais de saúde no desenvolvimento de suas funções e verificar o conhecimento destes acerca dos instrumentos de planejamento em saúde.

\section{Material e método}

Trata-se de uma pesquisa descritiva de abordagem qualitativa (MINAYO, 2013). Foram convidados a participar os 23 gestores (secretários municipais de saúde) de municípios da região de abrangência da $12^{a}$ Coordenadoria Regional de Saúde (CRS). No primeiro momento, foi contatado o coordenador regional de saúde com o intuito de apresentar o projeto e obter a declaração da entidade coparticipante. A pesquisadora participou de três reuniões da $12^{\circ} \mathrm{CRS}$, sendo uma para apresentar a pesquisa oferecer o Termo de Consentimento Livre e Esclarecido (TCLE) e disponibilizar o questionário para 
os participantes que demonstrassem interesse. Nas demais reuniões, foram recolhidos os TCLE e os questionários preenchidos.

Dos 23 gestores convidados, 10 aceitaram participar do estudo. A fim de manter o sigilo e anonimato dos participantes, estes foram identificados pela letra $G$ de gestor, seguida do número arábico correspondente à ordem de entrega do instrumento de coleta de dados e TCLE $\left(G_{1}, G_{2}, G_{3} \ldots G_{10}\right)$.

A análise dos dados foi realizada mediante verificação do conteúdo das falas dos participantes (MINAYO, 2015) pelo método de análise temática. Após a imersão e leitura dos resultados obtidos, os dados foram categorizados em duas categorias temáticas: dificuldades na gestão e planejamento das ações em saúde.

Destaca-se que em todas as etapas da pesquisa foram respeitados os aspectos éticos e legais vigentes para a pesquisa envolvendo seres humanos, estabelecidas pela Resolução 466/2012. 0 projeto de pesquisa foi aprovado pelo Comitê de Ética em Pesquisa do Instituto Federal Farroupilha, sob CAAE 80547717.9.0000.5574.

\section{Resultados e discussão}

Dos dez participantes, cinco já haviam exercido a atividade de gestão. Três já atuaram na própria secretaria de saúde, um teve experiência na gestão hospitalar e outro na secretaria municipal de educação. A seguir serão expostas as categorias obtidas no estudo bem como será realizada a discussão com a literatura.

\subsection{Dificuldades na gestão}

A complexidade da gerência é também um problema crônico enfrentado na gestão em saúde. Muitas vezes, essa dificuldade é atribuída a baixos salários, à rigidez e ao centralismo da administração pública (MARTINS; WACLAWOVSKY, 2015). A gestão em saúde é considerada como um dos grandes desafios para a consolidação do SUS e, muitas vezes, é direcionado aos interesses privados, com decisões voltadas às corporações, de médicos ou de outros profissionais em detrimento dos interesses e necessidades de saúde da população (CUNHA; HORTALE, 2017).

Uma equipe comprometida com o trabalho coletivo pode ser muito benéfica aos usuários dos serviços de saúde. No entanto, os gestores apontam o trabalho em equipe como uma das dificuldades encontradas no cotidiano gerencial:

[...] minha maior dificuldade está no gerenciamento do trabalho intrapessoal. $\left(\mathbf{G}_{1}\right)$

[...] há muito desgaste pela falta de comprometimento da maioria dos servidores da secretaria. $\left(\mathbf{G}_{2}\right)$

[...] o que dificulta muito o trabalho do gestor são as burocracias do serviço público e a gestão das equipes. $\left(\mathbf{G}_{3}\right)$

Em relação ao trabalho em equipe multiprofissional, é necessário que a equipe tenha conhecimento e valorize o trabalho do outro, de maneira que se construam conformidades no que se refere aos objetivos a serem atingidos, bem como o modo mais adequado ao se chegar à resolução do problema que se apresenta. Também é importante que os serviços disponham de estruturas adequadas, materiais e equipamentos, número adequado de profissionais para que seja oferecida uma assistência de qualidade e resolutiva. Além disso, o trabalho na área da saúde deve ser desenvolvido de forma coletiva, tratando-se de um desafio para a gestão em saúde. Isto exige práticas multi e interdisciplinares, de acordo com as necessidades do paciente. Assim sendo, se trata de um trabalho permeado por questões referentes às relações entre estes sujeitos e pela história das profissões em saúde (MARTINS; WACLAWOVSKY, 2015). 
O local de trabalho deve ter boas condições para o exercício da atividade, satisfazendo não só a prática da profissão em si, mas o bem-estar do funcionário, fornecendo a boa qualidade de vida no local de trabalho. Caso ele não encontre um ambiente de trabalho em condições adequadas, há a possibilidade de dano a todo corpo produtivo, uma vez que este poderá vir a desfalcar sua equipe que terá que se remanejar para cobrir essa falta, sobrecarregando o outro. O gestor deve se preocupar, além de zelar pelo ambiente de trabalho, assim como deve manter o servidor motivado, saudável e feliz, para que bons resultados possam ser obtidos. Se a equipe estiver estimulada a dar o melhor de si, os resultados alcançados serão melhores e consequentemente a população terá uma assistência de qualidade.

A burocracia e a formalidade excessivas são inimigas da equidade e da eficiência em Saúde Pública, pois, a complexidade da área acaba por ficar engessada frente aos inúmeros protocolos. De acordo com alguns autores, a burocratização e homogeneização emergem, sobretudo, fora do campo estrutural da organização (CUNHA; HORTALE, 2017; COSTA; NEVES, 2013). No entanto, o excesso burocrático pode travar processos, tendo como consequência o descumprimento do planejamento e a demora na distribuição de insumos, trazendo prejuízos aos usuários (BRASIL, 2019).

Uma das ferramentas na administração de finanças do governo é o orçamento, que se caracteriza como uma ação administrativa através do qual o Poder Legislativo autoriza o Poder Executivo a fazer alguma despesa pública, com o intuito de cobrir as despesas ao Estado ou a seguir a política econômica do País (RIBEIRO, 2017). No entanto, esse instrumento de planejamento deve ser usado pelo gestor com sabedoria e moderadamente, com o intuito de promover o bem-estar da população com os recursos disponíveis.

Dessa forma, o gestor público de saúde tem inúmeros compromissos como planejar, organizar, controlar, gerenciar a política de recursos humanos e de financiamento em âmbito local e regional, entre outros. Com relação aos recursos foi relatado:

0 trabalho na gestão da saúde do município é muito difícil porque temos uma sobrecarga de obrigações frente à falta de recursos disponíveis. $\left(\mathbf{G}_{4}\right)$

A sobrecarga no trabalho pode provocar cansaço físico e mental, baixa autoestima, desencadeando esgotamento emocional da pessoa, levando à chamada Síndrome de Burnout (POLLETO et al., 2016). Nesse sentido, o trabalho do gestor de saúde é difícil e desgastante, pois tem de um lado o usuário e suas demandas de saúde e, do outro, seus limites com relação ao orçamento, dificuldades nas relações com os funcionários, resultando em estresse, que não o deixa incapacitado para o trabalho, mas pode prejudicar o seu rendimento, deixando de realizar suas atividades a contento.

\subsection{Planejamento das ações em saúde}

O planejamento e instrumentos resultantes de seu processo, como planos e relatórios, é objeto de grande parte do arcabouço legal do SUS, seja apontando processos e métodos de formulação, seja como requisitos para fins de repasse de recursos, de controle e auditoria. As Leis $n^{\circ} 8.080 / 1990$ e $n^{\circ}$ 8.142/1990 (BRASIL, 1990; BRASIL 1990a) legalizam o planejamento e seus instrumentos. A primeira atribui à direção nacional do SUS a responsabilidade de elaborar o planejamento estratégico nacional no âmbito do SUS em cooperação com os estados, municípios e o Distrito Federal (BRASIL, 2009). Da mesma forma, destaca que o processo de planejamento e orçamento do SUS será ascendente, do nível local até o federal, ouvidos seus órgãos deliberativos, compatibilizando-se as necessidades da política de saúde com a disponibilidade de recursos em planos de saúde dos municípios, dos estados, do Distrito Federal e da União (BRASIL, 2009).

A formulação ascendente é um dos mecanismos importantes do princípio de unicidade do SUS. O seu cumprimento é desafio que tem que levar em conta as peculiaridades e necessidades próprias de 
cada município, estado e região do país, o que dificulta um modelo único que poderia ser aplicável em todas as instâncias. As falas a seguir expressam a percepção dos gestores em relação ao planejamento: [...] facilita o cumprimento da lei e planejamento para podermos coordenar a gestão. $\left(\mathbf{G}_{5}\right)$ Tenho amplo conhecimento sobre planejamento em conjunto com a equipe de apoio da gestão em saúde. $\left(\mathbf{G}_{3}\right)$

O planejamento regular mune os gestores de informações que permitem uma ação mais efetiva sobre a realidade sanitária e um redirecionamento das ações com o intuito de melhorar as condições de saúde da população. Contribui para a qualidade de vida das pessoas, através da prestação de ações e serviços de saúde adequados, resolutivos e humanizados, sendo essencial na gestão do SUS.

No entanto, são muitos os desafios que os governos municipais têm para atender às demandas por atenção e oferta de serviços. Nesse sentido, nem todos os gestores têm clareza sobre o planejamento, como pode ser observado nas falas a seguir:

Tenho pouco conhecimento sobre o planejamento. $\left(\mathbf{G}_{\mathbf{6}}\right)$

Desconheço em grande parte mas vejo que cada dia estou aprendendo mais. $\left(\mathbf{G}_{\mathbf{2}}\right)$

O Ministério da Saúde e as Secretarias Estaduais de Saúde recebem frequentemente alguns gestores e técnicos do SUS, com o intuito de fornecer orientações e cooperação técnica para a elaboração de instrumentos de planejamento, em especial planos de saúde e relatórios de gestão. Isso mostra que estados e municípios têm se esforçado para formulá-los, quer para fins de habilitação em uma condição de gestão (BRASIL, 2009).

Os instrumentos de planejamento da saúde incluem o plano de saúde e suas respectivas programações anuais e relatórios de gestão. No que se refere à política de saúde, a elaboração dos instrumentos de planejamento de governo deve observar o Plano Plurianual, Lei de Diretrizes Orçamentárias e Lei Orçamentária Anual, definidos a partir do artigo 165 da Constituição Federal (BRASIL, 2016). Sobre isso os gestores apontaram:

Conheço o Plano de Saúde e as programações anuais. $\left(\mathbf{G}_{\mathbf{7}}\right)$

Ainda estou estudando para aprender. $\left(\mathbf{G}_{\mathbf{2}}\right)$

Os Planos de Saúde têm vigência de quatro anos, e é fundamental a realização de sua avaliação, no final de seu cumprimento, deve ser relatada em documento que retrate os resultados efetivamente alcançados, capaz de subsidiar a elaboração do novo Plano (BRASIL, 2009). No entanto, percebe-se que nem todos os gestores estão afinados ou preparados aos instrumentos de planejamento e, portanto, não apresentam condições efetivas para administrar de maneira eficaz os recursos. Nesse sentido, é pertinente que os gestores busquem capacitações e ajuda através da valorização do conhecimento acumulado pelo conjunto de funcionários que dão continuidade às ações e serviços, independentemente das mudanças eleitorais, pois, a gestão do SUS é muito complexa e é imprescindível o diálogo democrático sobre os serviços e espaços.

Os Relatórios Anuais de Gestão devem, além de contemplar aspectos qualitativos e quantitativos, envolver também uma análise do processo geral de desenvolvimento do Plano, verificando os avanços obtidos, os obstáculos que dificultaram o trabalho, bem como as iniciativas ou medidas que devem ser desencadeadas (BRASIL, 2009). 


\section{Considerações finais}

No presente estudo, entre as dificuldades encontradas pelos gestores municipais de saúde no desenvolvimento de suas funções estão as relações com a equipe de trabalho, os aspectos burocráticos do serviço público e a sobrecarga de obrigações frente aos diminutos recursos financeiros disponíveis.

Quanto ao conhecimento dos gestores acerca dos instrumentos de planejamento em saúde, foi possível identificar que mesmo os participantes reconhecendo a importância destes, há ainda um grande desconhecimento sobre sua utilização na gestão em saúde. Assim, nem todos os gestores estão preparados para utilizar ou não conhecem suficientemente os instrumentos de planejamento e, portanto, não apresentam condições efetivas para administrar de maneira eficaz os recursos e planejar ações capazes de alterar positivamente a realidade de saúde da população de seus municípios.

Nesse sentido, o presente estudo traz como contribuição para a prática a identificação das fragilidades no que se refere ao conhecimento da gestão do sistema municipal de saúde dos gestores participantes do estudo, o que demonstra a necessidade de que que os gestores e futuros gestores passem por processos de capacitação e educação continuada a fim de que possam exercer suas atividades com maior fluidez e êxito, contribuindo, em última análise, para a melhoria da situação de saúde da população de seus municípios.

Destaca-se aqui que entre as limitações do presente estudo, está o número reduzido de participantes, o qual contemplou parcela inferior à metade da amostra total, bem como a condução do presente em uma única regional de saúde do estado do Rio Grande do Sul, resultando em uma realidade que poderá não refletir a de outras regionais do estado bem como do país.

\section{Referências}

BRASIL. BRASIL. [Constituição (1988)]. Constituição da República Federativa do Brasil. Brasília: Senado Federal, Coordenação de Edições técnicas, 2016. 496 p. Disponível em: https://www2.senado.leg.br/bdsf/ bitstream/handle/id/518231/CF88_Livro_EC91_2016.pdf. Acesso em: 23 de set. 2019.

BRASIL. Conselho Nacional de Secretarias Municipais de Saúde. Reflexões aos novos gestores municipais de saúde 2013-2016. Brasília: CONASEMS; 2013.

BRASIL. Ministério da Saúde. Sistema de Planejamento do SUS (PlanejaSUS): uma construção coletiva: trajetória e orientações de operacionalização. Brasília, DF: Ministério da Saúde, 2009. Disponível em: http:/l bvsms.saude.gov.br/bvs/publicacoes/planejaSUS_livro_1a6.pdf. Acesso em: 20 set. 2019.

BRASIL. Ministério da Saúde. Portaria nº 648/GM/MS, de 28 de março de 2006. Aprova a Política Nacional de Atenção Básica, estabelecendo a revisão de diretrizes e normas para a organização da Atenção Básica para o Programa Saúde da Família (PSF) e o Programa Agentes Comunitários de Saúde (PACS). Diário Oficial da União: seção 1, Brasília, DF, ano 2006, n. 42 p. 70, 29 de mar. 2006.

BRASIL. Lei $\mathbf{n}^{\circ} \mathbf{8 . 0 8 0}$, de 19 de setembro de $\mathbf{1 9 9 0}$. Dispõe sobre as condições para a promoção, proteção e recuperação da saúde, a organização e o funcionamento dos serviços correspondentes e dá outras providências. Diário Oficial da República Federativa do Brasil. Brasília: 1990a.

BRASIL. Lei $\mathbf{n}^{\circ} \mathbf{8 . 1 4 2}$, de 28 de dezembro de $\mathbf{1 9 9 0}$. Dispõe sobre a participação da comunidade na gestão do Sistema Único de Saúde (SUS) e sobre as transferências intergovernamentais de recursos financeiros na área da saúde e dá outras providências. Diário Oficial da República Federativa do Brasil. Brasília: 1990b. 
CAMPOS, G.W.S. Um método para análise e cogestão de coletivos: a constituição do sujeito, a produção de valor de uso e a democracia em instituições: o método da roda. São Paulo: Hucitec, 2000.

COSTA, L.A.; NEVES, J.A.B. Burocracia e inserção social: um estudo sobre o Ministério da Saúde na gestão do Sistema Único de Saúde. Saúde e Sociedade, v. 22, n.4, p. 1117-1131, 2013. Disponível em: http://www. scielo.br/scielo.php?pid=S0104-12902013000400014\&script=sci_abstract\&tlng=pt. Acesso em: 10 ago. 2019.

CUNHA, M.L.S; HORTALE, V.A. Características dos cursos voltados para a formação em gestão em saúde no Brasil. Saúde em Debate, v. 41, n. 113, p. 425-440, 2017. Disponível em: http://www.scielo.br/scielo. php?script=sci_arttext\&pid=S0103-11042017000200425\&lng=en\&nrm=isoAcesso em: 13 jun. 2019.

MARTINS, C.C.; WACLAWOVSKY, A.J. Problemas e desafios enfrentados pelos gestores públicos no processo de gestão em saúde. Gestão em Sistemas de Saúde, v. 4, n.1, p. 100-109, Jan/Jun. 2015. Disponível em: $<$ http://www.revistargss.org.br/ojs/index.php/rgss/article/view/157. Acesso em: 10 ago. 2019.

MINAYO, M.C.S. 0 desafio do conhecimento: pesquisa qualitativa em saúde. 13. ed. São Paulo: Hucitec, 2013.

POLLETO, N.A.; PROBST, L.F.; OLIVEIRA, T.L.; GUERRA, L.M.; AMBROSANO, G.M.B.; CORTELLAZZI, K.L. et al. Síndrome de Burnout em gestores municipais da saúde. Cadernos de Saúde Coletiva, v. 24, n. 2, p. 209-215, 2016. Disponível em: http://www.scielo.br/pdf/cadsc/v24n2/1414-462X-cadsc-24-2-209. pdf. Acesso em: 28 jul. 2019.

RIBEIRO, R.R.M. Orçamento público da saúde: um estudo do ciclo orçamentário no município de Maringá-PR. (Tese de Doutorado). São Paulo: Fundação Getúlio Vargas; 2017. 